\title{
Lightness induction enhancements and limitations at low frequency modulations across a variety of stimulus contexts
}

\author{
Louis Nicholas Vinke ${ }^{1,2}$, Arash Yazdanbakhsh ${ }^{\text {Corresp. 1, 2, } 3}$ \\ ${ }^{1}$ Graduate Program for Neuroscience, Boston University, Boston, United States \\ 2 Center for Systems Neuroscience (CSN), Boston University, Boston, United States \\ 3 Department of Psychological and Brain Sciences, Boston University, Boston, United States \\ Corresponding Author: Arash Yazdanbakhsh \\ Email address: yazdan@bu.edu
}

Lightness illusions are often studied under static viewing conditions with figures varying in geometric design, containing different types of perceptual grouping and figure-ground cues. A few studies have explored the perception of lightness induction while modulating lightness illusions continuously in time, where changes in perceived lightness are often linked to the temporal modulation frequency, up to around 2 - $4 \mathrm{~Hz}$. These findings support the concept of a cut-off frequency for lightness induction. However, another critical change (enhancement) in the magnitude of perceived lightness during slower temporal modulation conditions has not been addressed in previous temporal modulation studies. Moreover, it remains unclear whether this critical change applies to a variety of lightness illusion stimuli, and the degree to which different stimulus configurations can demonstrate enhanced lightness induction in low modulation frequencies. Therefore, we measured lightness induction strength by having participants cancel out any perceived modulation in lightness detected over time within a central target region, while the surrounding context, which ultimately drives the lightness illusion, was viewed in a static state or modulated continuously in time over a low frequency range $(0.25$ to $2 \mathrm{~Hz}$ ). In general, lightness induction decreased as temporal modulation frequency was increased, with the strongest perceived lightness induction occurring at lower modulation frequencies for visual illusions with strong grouping and figure-ground cues. When compared to static viewing conditions, we found that slow continuous surround modulation induces a strong and significant increase in perceived lightness for multiple types of lightness induction stimuli. Stimuli with perceptually ambiguous grouping and figure-ground cues showed weaker effects of slow modulation lightness enhancement. Our results demonstrate that, in addition to the existence of a cut-off frequency, an additional critical temporal modulation frequency of lightness induction exists $(0.25$ to $0.5 \mathrm{~Hz}$ ), which instead maximally enhances lightness induction and seems to be contingent upon the prevalence of figure-ground and grouping 
organization. 
1

2

3

4

5

6

7

8

9

10

11

12

13

14

15

16

17

18

19

20

21

22

23

24

25

26 Corresponding Author:

27 Arash Yazdanbakhsh ${ }^{1,2,3}$

28677 Beacon St., Boston, MA, 02215, USA

29 Email address: yazdan@bu.edu 


\section{Abstract}

31 Lightness illusions are often studied under static viewing conditions with figures varying in geometric design, containing different types of perceptual grouping and figure-ground cues. A few studies have explored the perception of lightness induction while modulating lightness illusions continuously in time, where changes in perceived lightness are often linked to the temporal modulation frequency, up to around $2-4 \mathrm{~Hz}$. These findings support the concept of a cut-off frequency for lightness induction. However, another critical change (enhancement) in the magnitude of perceived lightness during slower temporal modulation conditions has not been addressed in previous temporal modulation studies. Moreover, it remains unclear whether this critical change applies to a variety of lightness illusion stimuli, and the degree to which different stimulus configurations can demonstrate enhanced lightness induction in low modulation frequencies. Therefore, we measured lightness induction strength by having participants cancel out any perceived modulation in lightness detected over time within a central target region, while the surrounding context, which ultimately drives the lightness illusion, was viewed in a static state or modulated continuously in time over a low frequency range $(0.25$ to $2 \mathrm{~Hz})$. In general, lightness induction decreased as temporal modulation frequency was increased, with the strongest perceived lightness induction occurring at lower modulation frequencies for visual illusions with strong grouping and figure-ground cues. When compared to static viewing conditions, we found that slow continuous surround modulation induces a strong and significant increase in perceived lightness for multiple types of lightness induction stimuli. Stimuli with perceptually ambiguous grouping and figure-ground cues showed weaker effects of slow modulation lightness enhancement. Our results demonstrate that, in addition to the existence of a cut-off frequency, an additional critical temporal modulation frequency of lightness induction exists $(0.25$ to $0.5 \mathrm{~Hz})$, which instead maximally enhances lightness induction and seems to be contingent upon the prevalence of figureground and grouping organization. 


\section{Introduction}

63

64 65 66

Visual illusions which induce illusory lightness percepts of a central area with uniform reflectance are highly dependent upon the static luminance profile of adjacent components in the stimulus (Kingdom, 1997). These types of illusions have been mostly studied under static viewing conditions (Blakeslee \& McCourt, 1999; Heinemann, 1955; White, 1979; Williams, McCoy, \& Purves, 1998), wherein the perceived lightness is compared across various static contexts. Few studies have explored the perception of lightness induction illusions while modulating lightness illusions continuously in time (De Valois, Webster, De Valois, \& Lingelbach, 1986; Penacchio, Otazu, \& Dempere-Marco, 2013; Rossi \& Paradiso, 1996; 1999). When certain components of these illusions are modulated in time, human observers often report induced lightness modulation occurring within other components containing a static uniform luminance and reflectance, which is linked to the current modulation frequency. The temporal modulation of lightness induction has been shown to be a relatively slow process, operating up until 2 - 4 Hz (De Valois et al., 1986; Rossi \& Paradiso, 1996), after which a flickering percept may persist, followed by a complete loss of lightness induction as the modulation frequency is further increased. However, alternative experimental paradigms support the existence of a much faster process operating up to at least 24 $\mathrm{Hz}$ (Blakeslee \& McCourt, 2008).

An under-appreciated result observed in these few continuous lightness modulation studies is a relatively large increase in lightness induction when simply comparing static and slow continuous modulation viewing conditions (Rossi \& Paradiso, 1996; 1999), which in one instance did not actually bear out in the results, despite the authors anecdotal observations (De Valois et al., 1986). While much attention has been focused on cut-off frequency states at higher temporal modulation frequencies, currently no studies have explicitly explored the potential existence for another critical lightness induction state change at very low temporal modulation regimes $(<0.5 \mathrm{~Hz})$. The possibility of a significant change in lightness induction, by simply introducing a relatively slow continuous modulation, remains poorly understood and requires a thorough investigation.

Various types of visual illusions in a static context have been studied using a perceptual grouping framework, which is largely based upon Gestalt grouping principles (Wagemans, Elder, Kubovy, Palmer, Peterson, Singh, Heydt von der, 2012). For lightness illusions, grouping principles, such as generalized common fate, element connectedness, and uniform connectedness, 
92 can be considered to be the primary driving forces behind many perceptual illusions (Gilchrist, 93 2014). With the introduction of temporal modulation, common temporal structure and an alternate 94 interpretation of the generalized common fate principle have also been considered (Guttman, 95 Gilroy, \& Blake, 2007; Lee \& Blake, 1999; Sekuler \& Bennett, 2001). Lightness illusions have 96 also been differentiated based on the figure-ground cues present at border junctions, which are 97 thought to provide local occlusion cues to the observer (Anderson, 1997; Anderson, 2003). In this 98 case, different junction types are thought to facilitate the perception of depth relationships between 99 different objects in the scene. Common junction types include L-junctions (Simultaneous Contrast 100 illusion), T-junctions (Münker-White illusion), and X-junctions (Checkerboard illusion). The L101 and T-junctions are thought to provide strong cues for figure-ground segregation (Ross \& Pessoa, 102 2000; Todorović, 1997) by highlighting a shared border between two or more separate regions, 103 that is ultimately perceived as an occluding edge of the group of regions sharing that border. As a 104 result of border-ownership assignment, some regions are then more likely to be perceived as the 105 background, with the occluding region being the figure. This can occur despite retinal adjacency 106 of stimulus components under 2D viewing conditions (Layton, Mingolla, \& Yazdanbakhsh, 2012; 107 Zaidi, Spehar, \& Shy, 1997). Conversely, X- junctions tend to promote ambiguity between the 108 perceptual inferences of occlusion (multiple planes), adjacency (single plane), and/or transparency 109 (Adelson, 1993). While the dynamics that may be present in a visual illusion can be interpreted 110 using certain perceptual grouping principles, questions still remain regarding how different types 111 of figure-ground cues are affected by temporal dynamics, and the extent to which perceptual 112 grouping principles may reinforce these cues to accentuate lightness induction under continuous 113 temporal modulation viewing conditions relative to static viewing.

114 There are two main goals in the present study: (1) To explicitly examine whether lightness 115 induction undergoes any critical change between static viewing and continuous modulation states.

116 (2) To explore the extent to which different perceptual grouping principles and figure-ground cues 117 may contribute to any critical changes we observe across modulation conditions. While the visual 118 illusions examined in this study are differentiated based on figure-ground junction type, the results 119 are interpreted in terms of both perceptual grouping and figure-ground definitions. In general, 120 improving our understanding of how temporal modulation interacts with grouping and 121 segmentation cues applicable to a particular visual lightness illusion can offer further insight into 122 the early visuocortical processes involved in the percept of lightness illusions in humans. 
124 Materials \& Methods

125 Participants. Nine participants (3 female, ages 18 - 45) with normal or corrected-to-normal 126 vision were enrolled in this study. Participants were recruited from the Boston University 127 community. All participants received class credit or monetary compensation for their participation.

128 This study was approved by the Boston University Charles River Campus Institutional Review 129 Board (3651E). All participants were fully informed about the goals of the study and provided 130 written consent before their participation.

131 Stimuli. All stimuli were generated in MATLAB 2013b (TheMathWorks, 2012), using the 132 Psychophysics Toolbox (Brainard, 1997). A LCD monitor (Hanns-G Hi221; mean luminance: $13350.19 \mathrm{~cd} / \mathrm{m}^{2}$; frame rate: $60 \mathrm{~Hz}$ ) was used to display the stimuli, which was linearized using 134 gamma-correction generated from photometer measurements (Konica Minolta, LS-100). The 135 entire display region subtended $27.6^{\circ} \mathrm{x} 42.78^{\circ}$ (height $\mathrm{x}$ width) of the participant's visual angle. 136 All stimulus types are depicted in Figure 1A-D. The gray target region was located at central 137 fixation, and was $2.3^{\circ} \times 2.3^{\circ}$ (height $\mathrm{x}$ width) for all stimulus types. The dimensions for the 138 Simultaneous Contrast (SC), and Checkerboard (Chk) stimulus types was $6.9^{\circ}$ x $6.9^{\circ}$ (height $\mathrm{x}$ 139 width). The Münker-White stimulus type encompassed an assimilator $\left(\mathrm{MW}_{\mathrm{a}}\right)$ and a flanker $\left(\mathrm{MW}_{\mathrm{f}}\right)$ 140 subtype, each measuring $6.9^{\circ} \mathrm{x} 10.4^{\circ}$ (height $\mathrm{x}$ width). The dimensions of the Münker-White 141 stimuli were chosen such that the assimilator area matched the total area of the other stimulus 142 types. As a result, the flanker area was approximately one third the size of the assimilator area in 143 order to maintain the general Münker-White configuration and the square aspect ratio of the gray 144 target region.

145 Experimental Design and Procedure. Previous lightness induction studies have employed 146 paradigms where participants are asked to match the magnitude of the lightness illusion to a probe 147 (De Valois et al., 1986; Zaidi et al., 1997). This often proves to be a difficult task for participants, 148 as task difficulty tends to scale with increasing temporal frequency. To overcome the task difficulty 149 imposed by matching paradigms, in the present study participants were asked to perform a nulling 150 paradigm task (Krauskopf, Zaidi, \& Mandlert, 1986; Zaidi, Yoshimi, \& Flannigan, 1991; Adelson, 151 1993) when viewing each stimulus type under a variety of temporal modulation conditions. 152 Specifically, participants were asked to cancel out, as best as possible, any perceived modulation 153 in lightness detected over time within the central target region located at fixation. While carrying 
154 out the nulling task, the context provided by the surrounding lightness illusion stimulus either 155 alternated irregularly between two static states or was modulated continuously in time. Throughout 156 any given trial, participants adjusted the perceived 'grayness' of the central target region until they 157 had eliminated, as best as possible, any perceived differences in lightness over time (see Figure 158 1E). Additionally, participants were instructed to maintain central fixation throughout the entirety 159 of each trial (red fixation annulus: $0.15^{\circ}$ diameter). At the beginning of each trial, the central target 160 region was randomly initialized at either the mean luminance, matching the background, or at +/$16125 \%$ relative to the mean luminance. In-between each trial, subjects were presented with a $20 \mathrm{~Hz}$ 162 flickering random white noise whole-field patch for $500 \mathrm{~ms}$ to eliminate any persisting afterimage 163 related to stimuli viewed during the previous trial. Participants were given the opportunity to take 164 a break after each block of trials.

165 On continuous surround modulation (CSM) trials, the surrounding context oscillated 166 sinusoidally at one of several different logarithmically-spaced frequencies $(0.25,0.5,1$, and $2 \mathrm{~Hz})$. 167 The maximum and minimum luminance values of the dynamic stimulus segments during CSM 168 trials ranged between $90 \%$ and $10 \%$ of the maximum luminance, respectively, in order to maintain

169 physical borders between neighboring stimulus segments. For instance, this ensured that the $\mathrm{MW}_{\mathrm{a}}$ 170 and $\mathrm{MW}_{\mathrm{f}}$ stimuli could not be mistaken for the SC stimulus at these extreme states (Figure 1C-D).

171 Each block of CSM nulling trials consisted of 4 stimulus types (SC, Chk, MW

172 modulation frequencies $(0.25,0.5,1$, and $2 \mathrm{~Hz})$ repeated 4 times in a random order. Of the total 173 nine participants, six completed 1 block of CSM trials (64 trials in total), while the first initial three 174 participants completed 2 blocks of CSM trials (128 trials in total). An adequate number of frames 175 per continuous modulation cycle was achieved for all stimulus types $(0.25 \mathrm{~Hz}$ : 240 frames per 176 cycle; $2 \mathrm{~Hz}$ : 30 frames per cycle), resulting in no aliasing or perceived jitter in stimulus modulation, 177 ultimately creating a smooth appearance of sinusoidal modulation.

178 On static trials, participants had to manually switch between the stimulus context extremes, 179 which was subject to a forced 2 second delay in order to prevent participants from switching at a 180 frequency exceeding $0.25 \mathrm{~Hz}$. In practice, all participants switched at a much slower and irregular 181 rate. Each block of static nulling trials consisted of 3 stimulus types (SC, Chk, MW) repeated 4 182 times in a random order. Of the total nine participants, six completed 1 block of static trials (12 
183 trials in total), while the first initial three participants completed 2 blocks of static trials (24 trials 184 in total).

185 Since the static versions of the $\mathrm{MW}_{\mathrm{a}}$ and $\mathrm{MW}_{\mathrm{f}}$ stimuli were identical, only one set of static 186 nulling estimates were obtained for both of these stimulus types. This set of static nulling estimates

187 was used for comparison against both $\mathrm{MW}_{\mathrm{a}}$ and $\mathrm{MW}_{\mathrm{f}}$ nulling estimates obtained separately across 188 all the CSM conditions.

189 Data Analysis. The average nulling amplitude was calculated for all four stimulus types across 190 all five modulation conditions (static and continuous). Nulling amplitude, on any given trial, was 191 obtained as the final gray offset value of the central target region from mean luminance, and 192 calculated in percent difference relative to the mean display luminance. The nulling amplitude was 193 analogous to the percent difference from mean luminance, where a nulling amplitude of $0 \%$ 194 corresponded to a gray value of the central target region which was no different from the mean 195 display luminance. An intermediate nulling amplitude, e.g. 20\%, corresponded to a gray offset 196 value of the central target region which was maximally $20 \%$ lighter and darker than the mean 197 display luminance during modulation (static or continuous). Two-way between-subjects ANOVA 198 statistical tests were performed to determine whether any significant main effects and interactions 199 of stimulus type and temporal modulation on group-wise mean nulling amplitude measurements existed. Furthermore, to determine whether any significant trends existed across log-spaced modulation frequency conditions, a linear function was fit to the data, at the subject-wise level, using a least-squares fitting procedure. The Bonferroni method was used to correct for multiple 203 comparisons $(\mathrm{alpha}=0.0125)$ for all subsequent t-test statistical analyses.

\section{Results}

In order to investigate the combined effects of continuous surround modulation (CSM) frequency and segmentation cues on lightness induction, perceived lightness modulation induced in a central target region was measured across multiple modulation frequencies using a nulling paradigm. The results of a two-way between-subjects ANOVA (excluding the static condition) demonstrated a main effect of CSM frequency $\left(\mathrm{F}_{3,128}=7.73, \mathrm{p}<0.001\right)$, and a main effect of

211 stimulus type $\left(\mathrm{F}_{3,128}=11.19, \mathrm{p}<0.001\right)$. No significant interaction between CSM frequency and 
212 stimulus type was found $\left(\mathrm{F}_{9,128}=0.42, \mathrm{p}=0.923\right)$. In general, nulling amplitude decreased as

213 CSM frequency increased, indicating that the strongest perceived lightness induction occurred at

214 lower CSM frequencies (Figure 2A). For the slowest CSM frequency condition, the L-type

215 junction stimulus (SC) had the highest mean nulling amplitude $(22.6 \%+/-6.1)$, indicating this

216 stimulus type produced the strongest lightness induction perception. Stimuli containing T-type

217 junctions also produced relatively high mean nulling amplitudes $\left(\mathrm{MW}_{\mathrm{f}}: 19.8 \%+/-9.0 ; \mathrm{MW}_{\mathrm{a}}\right.$ :

218 18.1\% +/- 11.9). The X-type junction stimulus (Chk) had the lowest mean nulling amplitude

$219(10.1 \%+/-6.5)$, producing the weakest lightness induction, approximately half as strong as the L-

220 type junction stimulus (SC).

221 Lightness induction trends across continuous surround modulation frequencies. To further 222 examine the effect of CSM frequency on lightness induction, a linear function was fit to the mean 223 group nulling amplitude measurements for each stimulus condition across all CSM frequencies 224 converted to log-space. The slope parameter of the fitted linear function reflects any linear trend 225 in lightness induction across the log-spaced CSM frequencies (Figure 2B). Group-averaged slope 226 estimates were significantly different from zero for the L-type junction stimulus ( $\mathrm{SC}$ : [t(8) = 227 6.0297, $\mathrm{p}<.0 .001])$ and for both flanker $\left(\mathrm{MW}_{\mathrm{f}}:[\mathrm{t}(8)=-3.7663, \mathrm{p}=0.0055]\right)$ and assimilator $228\left(\mathrm{MW}_{\mathrm{a}}:[\mathrm{t}(8)=-2.4478, \mathrm{p}=0.0401]\right)$ T-type junction stimuli, while no significant difference was 229 found for the X-type junction stimulus (Chk: $[\mathrm{t}(8)=-1.4791, \mathrm{p}=0.1774]$ ). In general, all 230 significant group-averaged slope estimates were negative, reflecting a decrease in perceived 231 lightness induction as the CSM frequency was increased. These results indicate that the degree of 232 perceived lightness induction produced by L-type (SC) and both T-type $\left(\mathrm{MW}_{\mathrm{f}} \& \mathrm{MW}_{\mathrm{a}}\right)$ stimulus 233 configurations is particularly contingent upon CSM frequency.

234 Effect of continuous surround modulation vs. static viewing on lightness induction. The 235 degree to which even a slight degree of continuous surround temporal modulation can alter 236 lightness induction was examined by comparing the nulling amplitude responses between the static 237 condition and the slowest continuous surround modulation (CSM) condition $(0.25 \mathrm{~Hz}, 1$ cycle per 2384 seconds), for all stimulus types (Figure 3). Significant differences between perceived lightness 239 in the static and (slowest) CSM conditions were found for the L-type junction stimulus (SC: [t(8) $240=4.4485, \mathrm{p}=0.0021])$, and both T-type junction stimuli $\left(\mathrm{MW}_{\mathrm{f}}:[\mathrm{t}(8)=6.0532, \mathrm{p}<.0 .001] ; \mathrm{MW}_{\mathrm{a}}\right.$ : 
$241[\mathrm{t}(8)=3.5697, \mathrm{p}=0.0073])$. No significant difference was found for the X-type junction stimulus

242 (Chk: $[\mathrm{t}(8)=1.5204, \mathrm{p}=0.16])$. A comparison of effect sizes (Cohen's $d$ reported) indicates that

243 the flanker T-type junction stimulus had the greatest nulling amplitude difference between static

244 and the slowest dynamic modulation viewing conditions $\left(\mathrm{MW}_{\mathrm{f}}: d=2.2706\right)$, followed by the L-

245 type junction stimulus ( $\mathrm{SC}: d=1.7336)$, assimilator T-type junction stimulus $\left(\mathrm{MW}_{\mathrm{a}}: d=1.5619\right)$,

246 and lastly the X-type junction stimulus (Chk: $d=0.6291)$. These results strongly suggest that the

247 perceived lightness induction of stimuli containing figure-ground cues based only on L-type and

248 T-type junctions are augmented by slow $(0.25 \mathrm{~Hz})$ continuous surround temporal modulation.

\section{Discussion}

251 The findings and observations reported in previous studies examining the effects of temporal

252 modulation on the cut-off frequencies of perceived lightness indicate significant changes in 253 perceived lightness between static and very slow continuous modulation states when presenting 254 stimuli analogous to the Simultaneous Contrast illusion (see (De Valois et al., 1986): Discussion 255 \& Figure 4; (Rossi \& Paradiso, 1996): Figure 4; (Rossi \& Paradiso, 1999): Figure 9), indicating 256 another critical change is present. However, since this critical change was never the primary focus 257 of these studies, a more thorough examination did not follow, despite the ubiquity of this finding 258 in past temporal modulation studies. In this study, we used a nulling paradigm to measure the 259 lightness illusion strength when contextual surrounds, containing different types of segmentation 260 cues, were modulated continuously at multiple low frequencies (continuous modulation), or 261 compared against a no temporal modulation condition (static), to investigate factors driving the 262 significant changes in perceived lightness when little to no temporal modulation is present. We

263 found significant increases in the perceived lightness during continuous low-frequency surround 264 modulation compared to the static condition for illusions with contexts containing L-type junctions 265 (SC), and T-type junctions $\left(\mathrm{MW}_{\mathrm{f}}\right.$, and $\left.\mathrm{MW}_{\mathrm{a}}\right)$, with the exception of an illusion with X-type 266 junctions (Chk). When manipulating the frequency of the continuous surround modulation, 267 lightness illusion strength was found to be significantly higher compared to the static condition, 268 before decreasing across frequency conditions (from low to high) for SC, $\mathrm{MW}_{\mathrm{f}}$, and $\mathrm{MW}_{\mathrm{a}}$ stimuli, 269 while the Chk stimulus had no significant decreasing trends. The results also indicate that there 
270 exists a critical frequency greater than $0 \mathrm{~Hz}$ and less than or equal to $0.25 \mathrm{~Hz}$ (i.e., 1 cycle per 4

271 seconds) where the strength of the lightness illusions is strongest for $\mathrm{SC}, \mathrm{MW}_{\mathrm{f}}$, and $\mathrm{MW}_{\mathrm{a}}$ stimuli,

272 except in the case of the Chk stimulus. In general, this collection of findings suggests that dynamic

273 changes in perceived lightness are dependent upon, at least in part, how apparent the figure-ground

274 separation is perceived by the observer.

275 Critical continuous surround modulation frequency. In this study we have identified a critical

276 frequency located at a much lower continuous modulation frequency that differs from the cut-off

277 frequency previously reported in the literature. As the temporal modulation frequency decreases

278 and approaches a static state, the lightness induction strength increases. Conceivably, this effect

279 persists until the temporal modulation frequency is no longer discriminable from the static state,

280 which could be considered to be the Just Noticeable Temporal Modulation (JNTM) threshold. It

281 remains to be seen whether the maximal lightness induction strength occurs at the JNTM threshold.

282 Alternatively, lightness induction may conform to an inverted-U shape or reach a plateau between

$2830.25 \mathrm{~Hz}$ and the JNTM (Figure 4). Furthermore, upon confirming the existence of a JNTM

284 threshold, it may be possible to identify a global maximum, or the most optimal frequency for

285 producing the strongest modulation of lightness induction while keeping other factors fixed. In

286 general, at a temporal modulation of $0.25 \mathrm{~Hz}$ we observed the largest lightness induction

287 modulation, greater than that of the static condition for all stimuli, with the exception of the Chk

288 stimulus. Previous studies investigating interactions between temporal modulation and lightness

289 induction have tested surround contrast modulations with Michelson Contrasts up to 60\% (De

290 Valois et al., 1986) and 28.5\% (Rossi \& Paradiso, 1996). However, these previous studies did not

291 devote much discussion to the substantial subjective differences between static and continuous

292 temporal modulation conditions. The larger differences which we observed may in part be driven

293 by modulating stimuli containing much greater contrast levels (maximum Michelson Contrast:

$29490 \%)$.

295 Our results also demonstrate a decline in lightness induction strength as continuous surround 296 modulation frequency is increased, consistent with the presence of a cut-off frequency above $2 \mathrm{~Hz}$.

297 All lightness illusions we examined did show a significant ( $\mathrm{SC}, \mathrm{MW}_{\mathrm{f}}$, and $\mathrm{MW}_{\mathrm{a}}$ ) or trending

298 decline (Chk) in lightness induction strength as frequency increased. The effect of continuous

299 surround modulation does seem to impact the SC and MW stimuli in a qualitatively different way. 
300 The surround configuration of these stimuli may provide a stronger and unambiguous figure-

301 ground signal compared to the other stimuli, making the lightness induction more apparent despite

302 all stimuli sharing certain general geometric similarities. Conversely, the lightness induction

303 magnitude of the Chk stimulus seems to benefit the least from continuous surround modulation,

304 suggesting that this type of modulation does little to establish or improve the already weak figure-

305 ground visual cues present during the static viewing condition. Our results suggest that these

306 perceived lightness differences may be dependent upon the integration time of the stimulus

307 components at the cortical level. The variability in the effect of continuous surround modulation

308 on lightness induction across stimulus types may be related to the number of additional levels of

309 cortical processing necessary beyond the simple filtering properties of the retina and subcortical

310 visual areas (Jehee, Lamme, \& Roelfsema, 2007).

311 Extent of cortical recruitment for lightness induction. Evidence indicating that lightness

312 induction can operate over distances larger than conventional retinal and geniculate receptive

313 fields (De Valois \& Pease, 1971; Yund \& Armington, 1975) strongly suggests that lightness

314 induction requires cortical recruitment in order for the lightness induction percept to emerge. More

315 direct support for this claim comes from extracellular recordings, wherein striate cells were most

316 likely to display activity phase-locked to flanker modulation of the lightness induction stimulus,

317 despite the flanker being positioned outside of the conventional receptive field area (Rossi \&

318 Paradiso, 1996; 1999). Interestingly, when the central gray region undergoing lightness induction

319 is instead presented as uniformly black, then the phase-synchrony between striate activity and

320 flanker modulation is extinguished (Rossi \& Paradiso, 1999). This finding in particular suggests

321 that lightness induction either relies upon modulation from lateral connections within visuocortical

322 areas (Macknik \& Martinez-Conde, 2004), or relies upon modulation from inter-areal connections

323 across the visual hierarchy within the cortex (Angelucci et al., 2002; Bullier, 2001). In the latter

324 case, the entire induction stimulus (center and flanker components) can both be contained within

325 the larger conventional receptive field area in higher-order visual areas, and can be integrated as a

326 whole before being propagated back to earlier visuocortical areas where the lightness induction

327 phase-synchrony signature has been observed across all laminar layers (Rossi \& Paradiso, 1999).

328 Differences in edge-related single-unit activity coinciding with physical and illusory stimulus

329 contours have been reported across early visuocortical areas in non-human primates (Heydt,

330 Peterhans, \& Baumgartner, 1984; Zhou, Friedman, \& Heydt, 2000). However, a recent human 
331 fMRI study did not find distinct responses of neural populations representing the perceptual

332 lightness modulation of uniform stimulus regions, while still finding strong support for edge-

333 related responses (Cornelissen, Wade, Vladusich, Dougherty, \& Wandell, 2006).

334 The hierarchical recruitment of cortical areas during lightness perception has also inspired

335 modeling efforts which contain multiple banks of spatial filters at different orientations and spatial

336 scales (spatial frequency) (Blakeslee \& McCourt, 1999; Blakeslee \& McCourt, 2004; Dakin \&

337 Bex, 2001; Dakin \& Bex, 2003). These models have been shown to make quantitative predictions

338 for multiple visual illusions (Simultaneous Contrast, Münker-White, and others), which coincide

339 with perceptual judgments, although it has recently been shown that this class of models do not

340 capture the effect of narrowband noise on lightness induction (Betz, Shapley, Wichmann,

341 Maertens, 2015). Interestingly, Blakeslee \& McCourt, who have proposed and developed one of

342 these prominent models (ODOG model), have also provided evidence for temporal modulation

343 impacting brightness induction (grating induction stimulus), particularly at slow modulation

344 frequencies. One of the primary motivations behind these models is to support the notion of a fast

345 brightness induction process when perceiving these types of visual illusions, as opposed to

346 relatively slower filling-in mechanisms. However, the results of our study, and the complimentary

347 results of others, prompts the question of why more time for stimulus integration afforded during

348 slower temporal modulation leads to stronger illusion effects. While the addition of a temporal

349 component to the ODOG model has been suggested in order to capture these temporal modulation

350 effects (Blakeslee \& McCourt, 2011), recent implementations of the model have yet to include

351 temporal elements to capture dynamic changes in brightness induction (Blakeslee, Cope, \&

352 McCourt, 2016).

353 Lightness Illusion Differences. One previous study (Robinson, Hammon, \& de Sa, 2007) 354 reported the mean perceived lightness differences in luminance $\left(\mathrm{cd} / \mathrm{m}^{2}\right)$ from various lightness 355 illusion studies which used matching paradigms, with Simultaneous Contrast having the largest 356 effect $\left(11.35 \mathrm{~cd} / \mathrm{m}^{2}\right)$, followed by Checkerboard $\left(5.67 \mathrm{~cd} / \mathrm{m}^{2}\right)$, and Münker-White $\left(4.18 \mathrm{~cd} / \mathrm{m}^{2}\right)$. 357 Our results produced a slightly different ranking, with SC having the largest effect (static: 5.62 $358 \mathrm{~cd} / \mathrm{m}^{2} ; 0.25 \mathrm{~Hz}: 11.34 \mathrm{~cd} / \mathrm{m}^{2}$ ), followed by $\mathrm{MW}_{\mathrm{f}}$ (static: $2.21 \mathrm{~cd} / \mathrm{m}^{2} ; 0.25 \mathrm{~Hz}: 10.03 \mathrm{~cd} / \mathrm{m}^{2}$ ), $\mathrm{MW}_{\mathrm{a}}$ 359 (static: $2.21 \mathrm{~cd} / \mathrm{m}^{2} ; 0.25 \mathrm{~Hz}: 9.08 \mathrm{~cd} / \mathrm{m}^{2}$ ), and Chk (static: $3.71 \mathrm{~cd} / \mathrm{m}^{2} ; 0.25 \mathrm{~Hz}: 5.52 \mathrm{~cd} / \mathrm{m}^{2}$ ). It is 360 important to consider that absolute differences in lightness induction strengths between this study 361 and others could be accounted for by differences in stimulus spatial frequencies and stimulus size. 
362 Regarding stimulus size, the modulation area of each stimulus type was not always equated. When

363 all stimulus types are ranked by modulator area, the ranking roughly tracks the strength of the

364 illusions under static viewing conditions (see Figure 2A, static condition). However, when

365 temporal modulation is introduced, the correspondence is lost. Based on modulation area alone,

366 the $\mathrm{MW}_{\mathrm{f}}$ stimulus would be expected to show the smallest temporal modulation effect, but this is

367 not what we observed. The $\mathrm{MW}_{\mathrm{f}}$ nulling amplitude difference between the static and the lowest

368 frequency CSM condition was larger when compared to both the $\mathrm{MW}_{\mathrm{a}}$ and SC stimuli. Considering

369 just the MW stimuli, despite the smaller area of the flanker modulator compared to the assimilator,

370 the flanker modulator still generated a surprisingly strong lightness induction effect, which

371 suggests that if one were to control for modulator area, then the flanker effect may have been even

372 stronger. Relatedly, it has been reported that the lightness induction of the MW stimulus does not

373 depend on the aspect ratio of the target region, and hence the overall aspect ratio of the stimulus

374 (Blakeslee \& McCourt, 2004; Blakeslee, Padmanabhan, \& McCourt, 2016; Güçlü \& Farell, 2005),

375 but conflicting reports do exist (Mitra, Mazumdar, Ghosh, \& Bhaumik, 2018). Although, the

376 magnitude of the MW stimulus has been shown to be resilient across a wide range of lower spatial

377 frequencies, and demonstrates an increase across higher spatial frequencies (Blakeslee \& McCourt,

378 2004; Helson \& Rohles, 1959; White, 1979). It is also worth-noting that the aspect ratio of the SC

379 illusion has been shown to systematically influence lightness induction strength (Shi, Cui,

380 Troncoso, Macknik, \& Martinez-Conde, 2013; Yund \& Armington, 1975).

381 How might various perceptual grouping principles and figure-ground cues contribute to the

382 differences in lightness induction we observe across experimental conditions? While the

383 perceptual grouping principles mainly provide a guide for differentiating figural regions from

384 background regions, the grouping organization they promote identifies potential candidates for

385 figure or background designations. Some of the lightness illusions can adhere to the generalized

386 common fate principle (similarity in luminance) (Sekuler \& Bennett, 2001), and the uniform

387 connectedness principle (Palmer \& Rock, 1994). Considering the similarity principle, elements

388 with similar or identical luminance, will facilitate grouping for all three illusion types in this study,

389 with the mutually exclusive regions of each illusion having been initially identified based on local

390 uniform luminance properties (uniform connectedness). Unlike the MW illusion, the Chk illusion

391 does not benefit from the element connectedness principle, since none of the distinct uniform

392 regions which are grouped based on luminance similarities, share any continuous borders with one 
393 another. The lack of evidence for shared borders within each luminance group of the Chk illusion

394 is also conveyed with the figure-ground ambiguity associated with X-junctions. The flanker and

395 assimilator MW illusion types, differ in regards to grouping by element connectedness. The flanker

396 regions clearly share common (horizontal) occluding borders, the ownership of which is assigned

397 to the assimilator regions given the presence of the T-junctions, defining the assimilator regions

398 as figural components, and the flanker regions as background or as part of a different plane. Due

399 to the lack of multiple regions in the SC illusion (excluding the target region), the element

400 connectedness principle does not apply. Under temporal modulation conditions, a perceptual

401 grouping principle based on temporal structure comes online (Guttman, Gilroy, \& Blake, 2007;

402 Lee \& Blake, 1999). Simultaneous luminance changes in distinct regions occurring over time

403 further promotes the existing grouping organization already present under static viewing 404 conditions. Presumably, the temporal modulation of luminance does not have a direct impact on

405 the figure-ground organization supported by the different junction types, since the stimulus

406 component borders remain intact. However, our results demonstrate that temporal modulation

407 further reinforces the segregation of the figural and background groupings.

408 Nulling vs. Matching tasks. In contrast to previous studies using matching paradigms to 409 examine temporal effects on lightness illusions (De Valois et al., 1986; Rossi \& Paradiso, 1996;

410 Zaidi et al., 1997), we used a nulling paradigm which offers multiple advantages: (1) No

411 eccentricity confounds, (2) no short-term memory confounds, and (3) no covert attention demands.

412 Since subjects can perform the nulling procedure while maintaining fixation throughout the

413 task/trial, the perceived lightness measurements are being made at fixation relative to the

414 surrounding contexts, and generating no confounding issues of eccentricity or short-term memory.

415 Matching paradigms require participants to saccade back and forth between a target and matching 416 stimulus while remembering the 'grayness' in order to make their judgments, or alternatively, 417 participants covertly attend to a comparison target at a distal spatial location, often located several 418 degrees out from fixation. In this particular nulling paradigm, any potential confounds related to 419 the proximity of CSM frequencies to flicker fusion thresholds, wherein a rapidly changing stimulus 420 is perceived as having a steady appearance, are avoided since the highest CSM frequency measured $421(2 \mathrm{~Hz})$ is roughly two orders of magnitude lower than the flicker fusion threshold $(65-100 \mathrm{~Hz})$ 422 (Davis, Hsieh, \& Lee, 2015; Roberts \& Wilkins, 2013). Furthermore, while the mean background 423 luminance and spatial arrangement of each stimulus type was held constant across temporal 
424 modulation and static viewing conditions, the degree to which adaptation may have been perturbed 425 within each viewing condition may not be completely analogous due to the different modulation 426 changes being encountered. During static viewing, participants flipped between stimulus extremes 427 fairly regularly, at most every 2 seconds (imposed limit) up to every 3-4 seconds. Throughout 428 each trial participants were also systematically altering the target patch luminance between each 429 stimulus flip. These aperiodic stimulus modulations may have also perturbed the ability of 430 adaptation to any specific target gray level to take place, similar to modulation changes 431 encountered during the CSM conditions. However, it has been previously reported that following 432 sustained adaptation to a particular luminance level, the presentation time of probe stimuli over 433 1-4 seconds had minimal influence on apparent brightness judgments (Saunders, 1968).

434 Certain previous studies employing matching paradigms (De Valois et al., 1986) have noted 435 that some participants expressed difficulty with the matching task due to an asymmetry in the 436 lightness induction strength, specifically with a larger perceived induction strength as the 437 modulation approached the darkest state. Participants who experienced this asymmetry had 438 difficulty identifying a single value which matched the target. One participant in our experiments 439 reported a similar experience, indicating that this phenomenon can also apply to the nulling 440 paradigm. The prevalence of this phenomenon suggests that despite the fact that the temporal 441 modulation of the stimulus surround is centered at the mean luminance, which evenly bisects the 442 maximum and minimum surround luminance bounds, i.e. the two extreme states (black and white), 443 the percept of the illusion is not actually oscillating between equal perceptual bounds relative to 444 the neutral gray (i.e., mean display luminance). In other words, the balanced luminance extremes 445 imposed by the nulling procedure may not correspond to the extreme lightness induction states a 446 person actually perceives, which makes it difficult to identify and report a steady nulling level over 447 time. Recently, evidence from multiple studies have now shown that striate cells in non-human 448 primates actually respond more strongly to uniformly black stimuli than white, with the difference 449 being more prevalent at the stimulus center than at the edges (Kremkow, Jin, Komban, Wang, 450 Lashgari, Li, Jansen, Zaidi, \& Alonso, 2014; Xing, Yeh, \& Shapley, 2010; Zurawel, Ayzenshtat, 451 Zweig, Shapley, \& Slovin, 2014). Therefore, the assumption that both extreme static states of a 452 lightness illusion induce an equal lightness percept change (compared against a context-free 453 neutral gray) may not be true and requires further investigation. Any evidence for an asymmetry 454 in lightness induction toward lighter or darker percepts, despite the fact that the gray of the physical 
455 target is exactly in the middle of the maxima and minima, could reveal other perceptual biases of 456 the human visual system.

\section{Conclusions}

459 This study explicitly examined the existence of a critical change in lightness induction between static and continuous modulation states, while also exploring the extent to which different perceptual grouping principles and figure-ground cues alter the critical change of lightness induction in general within a low frequency modulation regime. Significant increases in lightness induction were found when comparing static against continuous surround modulation conditions for most stimulus types. Further increasing the continuous surround modulation frequency was also found to significantly decrease the lightness induction effect for certain stimulus types, while remaining unaltered for other stimulus types.

Study limitations. The fixed stimulus size and spatial frequency content in this study present a potential limitation. It is unclear if the results reported here under continuous modulation viewing conditions are resilient across these dimensions, or highly contingent upon them (Rossi \& Paradiso, 1996; Salmela \& Laurinen, 2009; Shi, Cui, Troncoso, Macknik, \& Martinez-Conde, 2013). Similarly, the luminance range over which the stimuli were manipulated is low and small relative to natural viewing conditions and scene statistics (Frazor \& Geisler, 2006; Radonjić, Allred, Gilchrist, \& Brainard, 2011). Whether or not low frequency lightness modulation persists across a wide range of absolute luminance spectrums remains to be investigated.

Future directions. The nulling paradigm employed in this study is easily transferable to a neuroimaging environment, whereby participants could be presented nulled (lightness induction absent) and non-nulled (lightness induction present) stimulus conditions. Population neural activity across early visuocortical areas could then be compared across continuous surround modulation frequency conditions to make inferences about the dependency of hierarchical cortical recruitment on the lightness perception and the strength of figure-ground cues and modulation frequency. Furthermore, it would be possible to differentiate sub-cortical and cortical areas which respond to either physical luminance or lightness induction modulation, or both, providing a better understanding of the emergence of lightness perception in humans. Lastly, there exist more geometrically complex lightness induction illusions, for example the Craik-O'Brien-Cornsweet 
486 be further studied under temporal modulation conditions. The large variety of feature induction

487 illusions, including color and contrast induction (Zaidi et al., 1997), which remain to be 488 investigated under static and continuous modulation presentation, could also offer further insight 489 into how humans perceive more complex objects and scenes.

490

491 Acknowledgements

492 We would like to thank the members of the Computational Neuroscience \& Vision lab for all 493 their help and support. The authors of this manuscript declare no conflicts of interest.

494

495 Figure Legends

496 Figure 1: Experimental Stimuli and Procedure.

497 Examples of lightness induction stimuli presented to subjects, and nulling task procedure. For all 498 experimental stimuli, the stimulus context (c) was temporally modulated while subjects adjusted 499 the central target $(\mathrm{t})$ region to eliminate any perceived lightness modulation. Two possible 500 extremes are depicted for each stimulus, each illustrating a pair of stimuli with the surround 501 inverted. (A) Simultaneous Contrast stimulus. (B) Checkerboard stimulus. (C) Münker-White 502 assimilator stimulus. Only the assimilator $\left(c_{a}\right)$ portions of the stimulus context were varied on 503 temporal modulation trials. Modulated components are depicted at a decreased contrast for

504 viewing purposes. (D) Münker-White flanker stimulus. Only the flanker $\left(\mathrm{c}_{\mathrm{f}}\right)$ portions of the

505 stimulus context were varied on temporal modulation trials. Modulated components are depicted 506 at a decreased contrast for viewing purposes. (E) Example time course of the simultaneous contrast 507 stimulus during the nulling procedure. At trial onset, the central target region had a fixed mean 508 luminance. However, subjects experienced an induced lightness modulation within the target 509 region, dependent on the temporal modulation frequency (dashed line). By adjusting the counter510 phase luminance offsets of the target region, subjects could reduce and eliminate any perceived

511 lightness modulation induced by stimulus context modulation, and effectively made the target 512 region appear as having a fixed mean luminance over time (solid line). The maximum and 513 minimum contrast for all 4 stimulus conditions were limited to $90 \%$ and $-90 \%$ respectively. 514

515 Figure 2: Effects of surround modulation and stimulus type on lightness induction. 
516 Group-wise $(n=9)$ effects and trends of temporal modulation frequency on nulling amplitudes

517 across all stimulus conditions. (A) Mean group nulling amplitude across the static and continuous

518 surround modulation frequency conditions for all stimulus types. A nulling amplitude of $0 \%$

519 would indicate no perceived lightness induction for that specific experimental condition and

520 stimulus type. (B) Mean group slope estimates representing any trends in nulling amplitude

521 measurements across the continuous surround modulation (CSM) frequency conditions. All error

522 bars represent one standard error of the mean (asterisks denote $\mathrm{p}<.0 .01$ ).

523

524 Figure 3: Effect of static vs. continuous modulation on lightness induction.

525 Group-wise effects of the static and continuous surround modulation conditions on lightness

526 induction across all stimulus conditions. Mean group nulling amplitude for the static condition

527 and the slowest continuous surround modulation (CSM) condition $(0.25 \mathrm{~Hz})$ across all 4 stimulus

528 types are depicted. An absolute nulling amplitude of $0 \%$ would indicate that no lightness

529 induction was being perceived, and the central target was physically identical to the mean

530 luminance background. Note that all static stimulus conditions have non-zero mean nulling

531 amplitudes, confirming previous observations that lightness induction does occur with these

532 particular surround organizations. The mean nulling amplitudes for both static Münker-White

533 flanker and assimilator stimuli are identical because they were measured and computed from the

534 identical trials (see Methods for details). All error bars represent one standard error of the mean

535 (asterisks denote $\mathrm{p}<.0 .01$ ).

536

\section{Figure 4: Just Noticeable Temporal Modulation (JNTM) limit}

538 Illustration depicting lightness induction strength trends as stimulus modulation frequency

539 approaches the limit at which continuous modulation of the stimulus can no longer be reliably

540 detected by the observer, and is perceived to be static in nature, termed the Just Noticeable

541 Temporal Modulation (JNTM) limit. Over increasingly slower temporal modulation frequencies,

542 the lightness induction strength is hypothesized to change in three qualitative ways: induction

543 strength will (a) continue to increase, (b) reach a plateau, or (c) monotonically decrease. An

544 inflection point (d) for the latter two hypothetical paths could occur at any point between the lowest

545 temporal modulation condition in this study $(0.25 \mathrm{~Hz})$ and the JNTM limit. Data points from the

546 Simultaneous Contrast condition are shown in low-opacity for illustrative purposes only. 
547 References

548 Adelson, E. H. (1993). Perceptual organization and the judgment of brightness. Science (New York, N.Y.), 262(5142), 2042-2044.

550

Anderson BL 1997. A Theory of Illusory Lightness and Transparency in Monocular and

551

552

Binocular Images: The Role of Contour Junctions. Perception 26:419-453. DOI:

553 10.1068/p260419.

Anderson BL 2003. Perceptual organization and White's illusion. Perception 32:269-284. DOI:

554 $10.1068 / \mathrm{p} 3216$.

Angelucci, A., Levitt, J. B., Walton, E. J. S., Hupé, J.-M., Bullier, J., \& Lund, J. S. (2002).

Circuits for Local and Global Signal Integration in Primary Visual Cortex. Journal of

Neuroscience, 22(19), 8633-8646. http://doi.org/10.1523/JNEUROSCI.22-19-08633.2002

558

559

560

561

562

563

564

565

566

567

568

569

570

571

572

573

574

575

576

577

Benary, W. (1924). Beobachtungen zu einem Experiment über Helligkeitskontrast.

Psychologische Forschung, 5(1), 131-142. http://doi.org/10.1007/BF00402398

Betz T, Shapley R, Wichmann FA, Maertens M 2015. Noise masking of White's illusion exposes the weakness of current spatial filtering models of lightness perception. Journal of Vision 15:1-17. DOI: 10.1167/15.14.1.

Blakeslee, B., \& McCourt, M. E. (1999). A multiscale spatial filtering account of the White effect, simultaneous brightness contrast and grating induction. Vision Research, 39(26), 4361-4377. http://doi.org/10.1016/S0042-6989(99)00119-4

Blakeslee B, McCourt ME 2004. A unified theory of brightness contrast and assimilation incorporating oriented multiscale spatial filtering and contrast normalization. Vision Research 44:2483-2503. DOI: 10.1016/j.visres.2004.05.015.

Blakeslee, B., \& McCourt, M. E. (2008). Nearly instantaneous brightness induction. Journal of Vision, 8(2), 15.1-8. http://doi.org/10.1167/8.2.15

Blakeslee B, McCourt ME 2011. Spatiotemporal analysis of brightness induction. Vision Research 51:1872-1879. DOI: 10.1016/j.visres.2011.06.018.

Blakeslee B, Cope D, McCourt ME 2016. The Oriented Difference of Gaussians (ODOG) model of brightness perception: Overview and executable Mathematica notebooks. Behavior research methods 48:306-312. DOI: 10.3758/s13428-015-0573-4.

Blakeslee B, Padmanabhan G, McCourt ME 2016. Dissecting the influence of the collinear and flanking bars in White's effect. Vision Research 127:11-17. DOI:

Peer] reviewing PDF | (2019:07:39538:1:1:NEW 1 Mar 2020) 
578

579

580

581

582

583

584

585

586

587

588

589

590

591

592

593

594

595

596

597

598

599

600

601

602

603

604

605

606

607

608

10.1016/j.visres.2016.07.001.

Brainard, D. H. (1997). The Psychophysics Toolbox. Spatial Vision, 10(4), 433-436. http://doi.org/10.1163/156856897X00357

Bullier, J. (2001). Integrated model of visual processing. Brain Research. Brain Research Reviews, 36(2-3), 96-107.

Cornelissen, F. W., Wade, A. R., Vladusich, T., Dougherty, R. F., \& Wandell, B. A. (2006). No functional magnetic resonance imaging evidence for brightness and color filling-in in early human visual cortex. The Journal of Neuroscience : the Official Journal of the Society for Neuroscience, 26(14), 3634-3641. http://doi.org/10.1523/JNEUROSCI.4382-05.2006

Dakin SC, Bex PJ 2001. Local and global visual grouping: tuning for spatial frequency and contrast. Journal of Vision 1:99-111. DOI: 10.1167/1.2.4.

Dakin SC, Bex PJ 2003. Natural image statistics mediate brightness "filling in." Proceedings of the Royal Society of London. Series B, Biological Sciences 270:2341-2348. DOI: 10.1098/rspb.2003.2528.

Davey, M. P., Maddess, T., \& Srinivasan, M. V. (1998). The spatiotemporal properties of the Craik-O'Brien-Cornsweet effect are consistent with “filling-in.” Vision Research, 38(13), 2037-2046. http://doi.org/10.1016/s0042-6989(97)00329-5

Davis, J., Hsieh, Y.-H., \& Lee, H.-C. (2015). Humans perceive flicker artifacts at 500 Hz. Nature Publishing Group, 5(1), 21-4. http://doi.org/10.1038/srep07861

De Valois, R. L., \& Pease, P. L. (1971). Contours and Contrast: Responses of Monkey Lateral Geniculate Nucleus Cells to Luminance and Color Figures. Science (New York, N.Y.), 171(3972), 694-696. http://doi.org/10.1126/science.171.3972.694

De Valois, R. L., Webster, M. A., De Valois, K. K., \& Lingelbach, B. (1986). Temporal properties of brightness and color induction. Vision Research, 26(6), 887-897.

Frazor, R. A., \& Geisler, W. S. (2006). Local luminance and contrast in natural images. Vision Research, 46(10), 1585-1598. http://doi.org/10.1016/j.visres.2005.06.038

Gilchrist A 2014. A Gestalt Account of Lightness Illusions. Perception 43:881-895. DOI: $10.1068 / \mathrm{p} 7751$.

Güçlü B, Farell B 2005. Influence of target size and luminance on the White-Todorović effect. Vision Research 45:1165-1176. DOI: 10.1016/j.visres.2004.10.025.

Guttman SE, Gilroy LA, Blake R 2007. Spatial grouping in human vision: Temporal structure 
609

610

611

612

613

614

615

616

617

618

619

620

621

622

623

624

625

626

627

628

629

630

631

632

633

634

635

636

637

638

639

trumps temporal synchrony. Vision Research 47:219-230. DOI:

10.1016/j.visres.2006.09.012.

Heinemann, E. G. (1955). Simultaneous brightness induction as a function of inducing and testfield luminances. Journal of Experimental Psychology, 50(2), 89-96.

Helson H, Rohles FH 1959. A quantitative study of reversal of classical lightness-contrast. The American journal of psychology 72:530-538.

Heydt, von der, R., Peterhans, E., \& Baumgartner, G. (1984). Illusory contours and cortical neuron responses. Science (New York, N.Y.), 224(4654), 1260-1262.

Jehee JFM, Lamme VAF, Roelfsema PR 2007. Boundary assignment in a recurrent network architecture. Vision Research 47:1153-1165. DOI: 10.1016/j.visres.2006.12.018.

Kingdom, F. (1997). Simultaneous contrast: the legacies of Hering and Helmholtz. Perception, 26(6), 673-677. http://doi.org/10.1068/p260673

Krauskopf J, Zaidi Q, Mandlert MB 1986. Mechanisms of simultaneous color induction. Journal of the Optical Society of America. A, Optics, image science, and vision 3:1752-7. DOI: 10.1364/JOSAA.3.001752.

Kremkow J, Jin J, Komban SJ, Wang Y, Lashgari R, Li X, Jansen M, Zaidi Q, Alonso J-M 2014. Neuronal nonlinearity explains greater visual spatial resolution for darks than lights. Proceedings of the National Academy of Sciences of the United States of America 111:3170-3175. DOI: 10.1073/pnas.1310442111.

Layton, O. W., Mingolla, E., \& Yazdanbakhsh, A. (2012). Dynamic coding of border-ownership in visual cortex. Journal of Vision, 12(13), 8-8. http://doi.org/10.1167/12.13.8

Lee SH, Blake R 1999. Visual form created solely from temporal structure. Science (New York, N.Y.) 284:1165-1168. DOI: 10.1126/science.284.5417.1165.

Macknik, S. L., \& Martinez-Conde, S. (2004). The spatial and temporal effects of lateral inhibitory networks and their relevance to the visibility of spatiotemporal edges. Neurocomputing, 58-60, 775-782. http://doi.org/10.1016/j.neucom.2004.01.126

Mitra S, Mazumdar D, Ghosh K, Bhaumik K 2018. An adaptive scale Gaussian filter to explain White's illusion from the viewpoint of lightness assimilation for a large range of variation in spatial frequency of the grating and aspect ratio of the targets. PeerJ 6:e5626. DOI: 10.7717/peerj.5626.

Palmer S, Rock I 1994. Rethinking perceptual organization: The role of uniform connectedness. 
640

641

642

643

644

645

646

647

648

649

650

651

652

653

654

655

656

657

658

659

660

661

662

663

664

665

666

667

668

669

670

Psychonomic Bulletin \& Review 1:29-55. DOI: 10.3758/BF03200760.

Penacchio, O., Otazu, X., \& Dempere-Marco, L. (2013). A neurodynamical model of brightness induction in v1. PloS One, 8(5), e64086. http://doi.org/10.1371/journal.pone.0064086

Radonjić, A., Allred, S. R., Gilchrist, A. L., \& Brainard, D. H. (2011). The Dynamic Range of Human Lightness Perception. Current Biology, 21(22), 1931-1936. http://doi.org/10.1016/j.cub.2011.10.013

Roberts, J. E., \& Wilkins, A. J. (2013). Flicker can be perceived during saccades at frequencies in excess of $1 \mathrm{kHz}$. Lighting Research \& Technology, 45(1), 124-132.

http://doi.org/10.1177/1477153512436367

Robinson, A. E., Hammon, P. S., \& de Sa, V. R. (2007). Explaining brightness illusions using spatial filtering and local response normalization. Vision Research, 47(12), 1631-1644. http://doi.org/10.1016/j.visres.2007.02.017

Ross WD, Pessoa L 2000. Lightness from contrast: a selective integration model. Perception \& psychophysics 62:1160-1181. DOI: 10.3758/bf03212120.

Rossi, A. F., \& Paradiso, M. A. (1996). Temporal limits of brightness induction and mechanisms of brightness perception. Vision Research, 36(10), 1391-1398.

Rossi, A. F., \& Paradiso, M. A. (1999). Neural correlates of perceived brightness in the retina, lateral geniculate nucleus, and striate cortex. The Journal of Neuroscience : the Official Journal of the Society for Neuroscience, 19(14), 6145-6156.

Salmela, V. R., \& Laurinen, P. I. (2009). Low-level features determine brightness in White's and Benary's illusions. Vision Research, 49(7), 682-690. http://doi.org/10.1016/j.visres.2009.01.006

Saunders JE 1968. Adaptation, its effect on apparent brightness and contribution to the phenomenon of brightness constancy. Vision Research 8:451-468. DOI: 10.1016/00426989(68)90113-2.

Sekuler AB, Bennett PJ 2001. Generalized Common Fate: Grouping by Common Luminance Changes. Psychological Science 12:437-444. DOI: 10.2307/40063667?refreqid=searchgateway:dd7e012d3a2641cd772ff26e437e1469.

Shi, V., Cui, J., Troncoso, X. G., Macknik, S. L., \& Martinez-Conde, S. (2013). Effect of stimulus width on simultaneous contrast. PeerJ, 1(5), e146-13.

http://doi.org/10.7717/peerj.146 
671 TheMathWorks, I. (2012). MATLAB and Statistics Toolbox Release 2012b. Natick.

672 Todorović D 1997. Lightness and junctions. Perception 26:379-394. DOI: 10.1068/p260379.

673 Wagemans J, Elder JH, Kubovy M, Palmer SE, Peterson MA, Singh M, Heydt von der R 2012.

674 A century of Gestalt psychology in visual perception: I. Perceptual grouping and figure-

675 ground organization. Psychological bulletin 138:1172-1217. DOI: 10.1037/a0029333.

676 White, M. (1979). A new effect of pattern on perceived lightness. Perception, 8(4), 413-416.

677 http://doi.org/10.1068/p080413

678 Williams, S. M., McCoy, A. N., \& Purves, D. (1998). The influence of depicted illumination on

679 brightness. Proceedings of the National Academy of Sciences of the United States of

680 America, 95(22), 13296-13300.

681 Xing D, Yeh C-I, Shapley RM 2010. Generation of black-dominant responses in V1 cortex. The 682 Journal of neuroscience : the official journal of the Society for Neuroscience 30:13504-

683 13512. DOI: 10.1523/JNEUROSCI.2473-10.2010.

684 Yund, E. W., \& Armington, J. C. (1975). Color and brightness contrast effects as a function of

685 spatial variables. Vision Research, 15(8-9), 917-929. http://doi.org/10.1016/0042-

686 6989(75)90231-X

687 Zaidi Q, Yoshimi B, Flannigan J 1991. Influence of shape and perimeter length on induced color 688 contrast. Journal of the Optical Society of America. A, Optics and image science 8:1810689 1817. DOI: $10.1364 /$ josaa.8.001810.

690 Zaidi, Q., Spehar, B., \& Shy, M. (1997). Induced effects of backgrounds and foregrounds in 691 three-dimensional configurations: the role of T-junctions. Perception, 26(4), 395-408.

692 http://doi.org/10.1068/p260395

693 Zhou, H., Friedman, H. S., \& Heydt, von der, R. (2000). Coding of border ownership in monkey 694 visual cortex. Journal of Neuroscience, 20(17), 6594-6611.

695 Zurawel G, Ayzenshtat I, Zweig S, Shapley R, Slovin H 2014. A contrast and surface code

696 explains complex responses to black and white stimuli in V1. The Journal of neuroscience :

697 the official journal of the Society for Neuroscience 34:14388-14402. DOI:

698 10.1523/JNEUROSCI.0848-14.2014.

699 


\section{Figure 1}

\section{Experimental Stimuli and Procedure.}

Examples of lightness induction stimuli presented to subjects, and nulling task procedure. For all experimental stimuli, the stimulus context (c) was temporally modulated while subjects adjusted the central target ( $\mathrm{t}$ ) region to eliminate any perceived lightness modulation. Two possible extremes are depicted for each stimulus, each illustrating a pair of stimuli with the surround inverted. (A) Simultaneous Contrast stimulus. (B) Checkerboard stimulus. (C) Münker-White assimilator stimulus. Only the assimilator $\left(C_{a}\right)$ portions of the stimulus context were varied on temporal modulation trials. Modulated components are depicted at a decreased contrast for viewing purposes. (D) Münker-White flanker stimulus. Only the flanker $\left(c_{f}\right)$ portions of the stimulus context were varied on temporal modulation trials. Modulated components are depicted at a decreased contrast for viewing purposes. (E) Example time course of the simultaneous contrast stimulus during the nulling procedure. At trial onset, the central target region had a fixed mean luminance. However, subjects experienced an induced lightness modulation within the target region, dependent on the temporal modulation frequency (dashed line). By adjusting the counter-phase luminance offsets of the target region, subjects could reduce and eliminate any perceived lightness modulation induced by stimulus context modulation, and effectively made the target region appear as having a fixed mean luminance over time (solid line). The maximum and minimum contrast for all 4 stimulus conditions were limited to $90 \%$ and $-90 \%$ respectively. 
A.
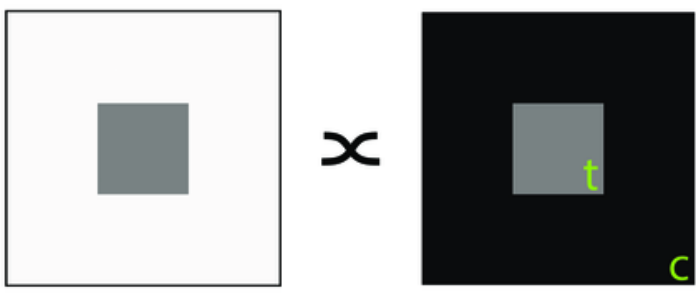

B.
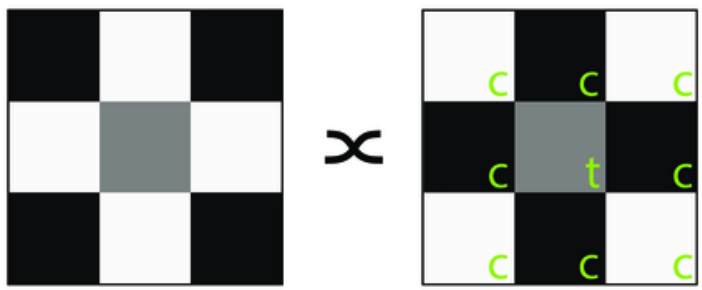

C.
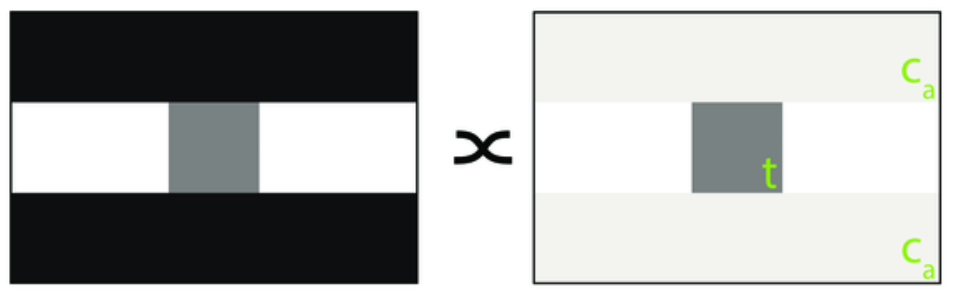

D.
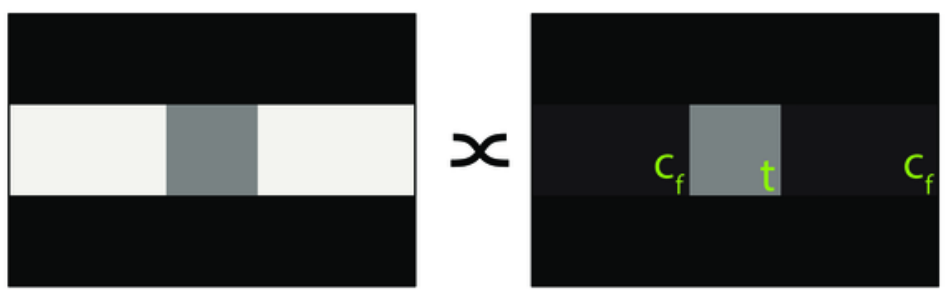

E.

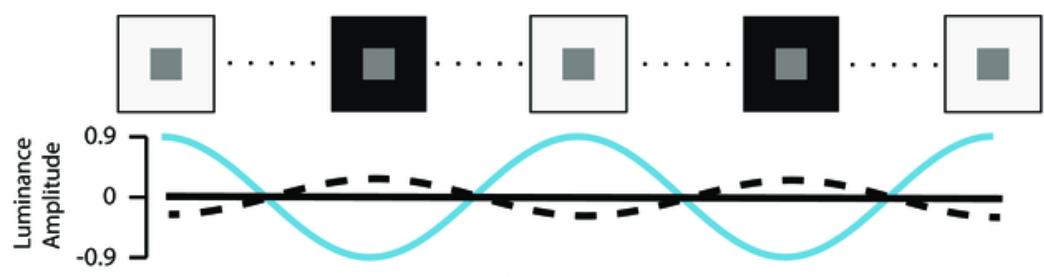

Luminance Inversion: $x$

- - Perceived Target Before Nulling Perceived Target After Nulling Surround Luminance Modulation 


\section{Figure 2}

Effects of surround modulation and stimulus type on lightness induction.

Group-wise $(n=9)$ effects and trends of temporal modulation frequency on nulling amplitudes across all stimulus conditions. (A) Mean group nulling amplitude across the static and continuous surround modulation frequency conditions for all stimulus types. A nulling amplitude of $0 \%$ would indicate no perceived lightness induction for that specific experimental condition and stimulus type. (B) Mean group slope estimates representing any trends in nulling amplitude measurements across the continuous surround modulation (CSM) frequency conditions. All error bars represent one standard error of the mean (asterisks denote $p<.0 .01)$.

A.

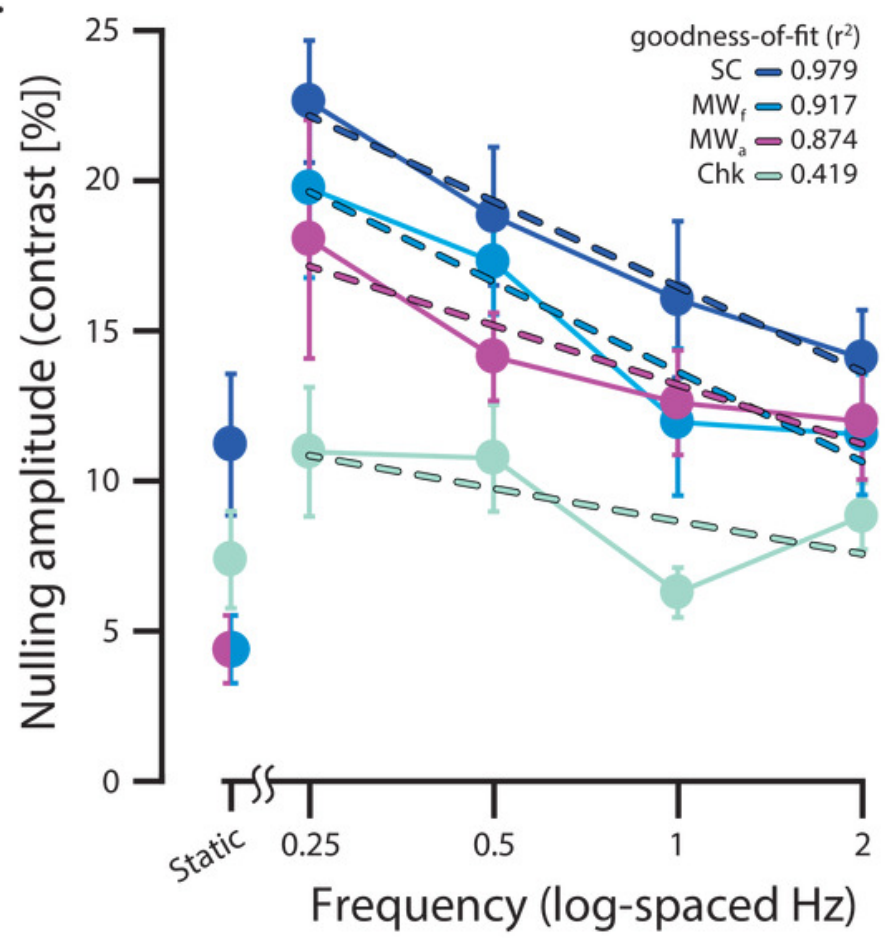

B.

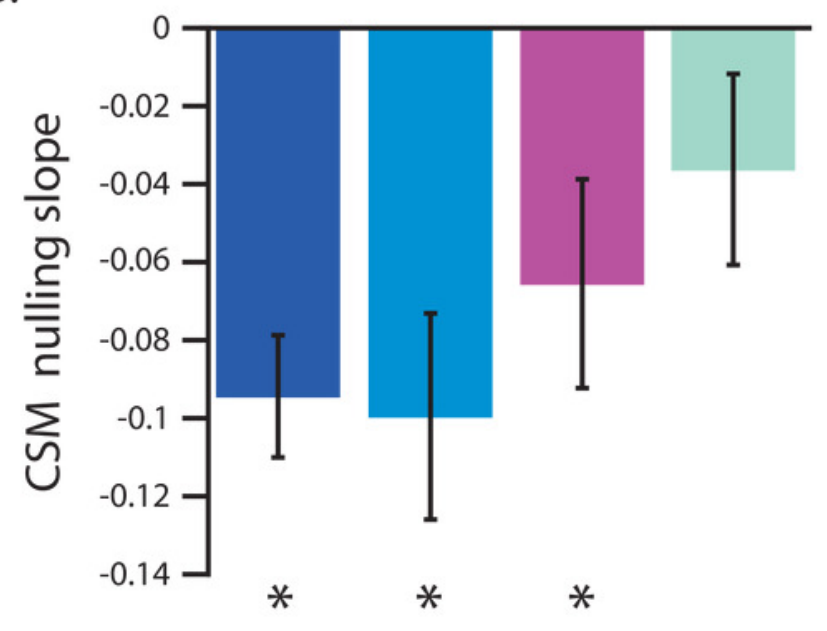

Simultaneous Contrast

Münker-White (flanker) Münker-White (assimilator) Checkerboard 


\section{Figure 3}

Effect of discrete vs. continuous modulation on lightness induction.

Group-wise effects of the static and continuous surround modulation conditions on lightness induction across all stimulus conditions. Mean group nulling amplitude for the static condition and the slowest continuous surround modulation (CSM) condition $(0.25 \mathrm{~Hz})$ across all 4 stimulus types are depicted. An absolute nulling amplitude of $0 \%$ would indicate that no lightness induction was being perceived, and the central target was physically identical to the mean luminance background. Note that all static stimulus conditions have non-zero mean nulling amplitudes, confirming previous observations that lightness induction does occur with these particular surround organizations. The mean nulling amplitudes for both static MünkerWhite flanker and assimilator stimuli are identical because they were measured and computed from the identical trials (see Methods for details). All error bars represent one standard error of the mean (asterisks denote $p<.0 .01$ ). 


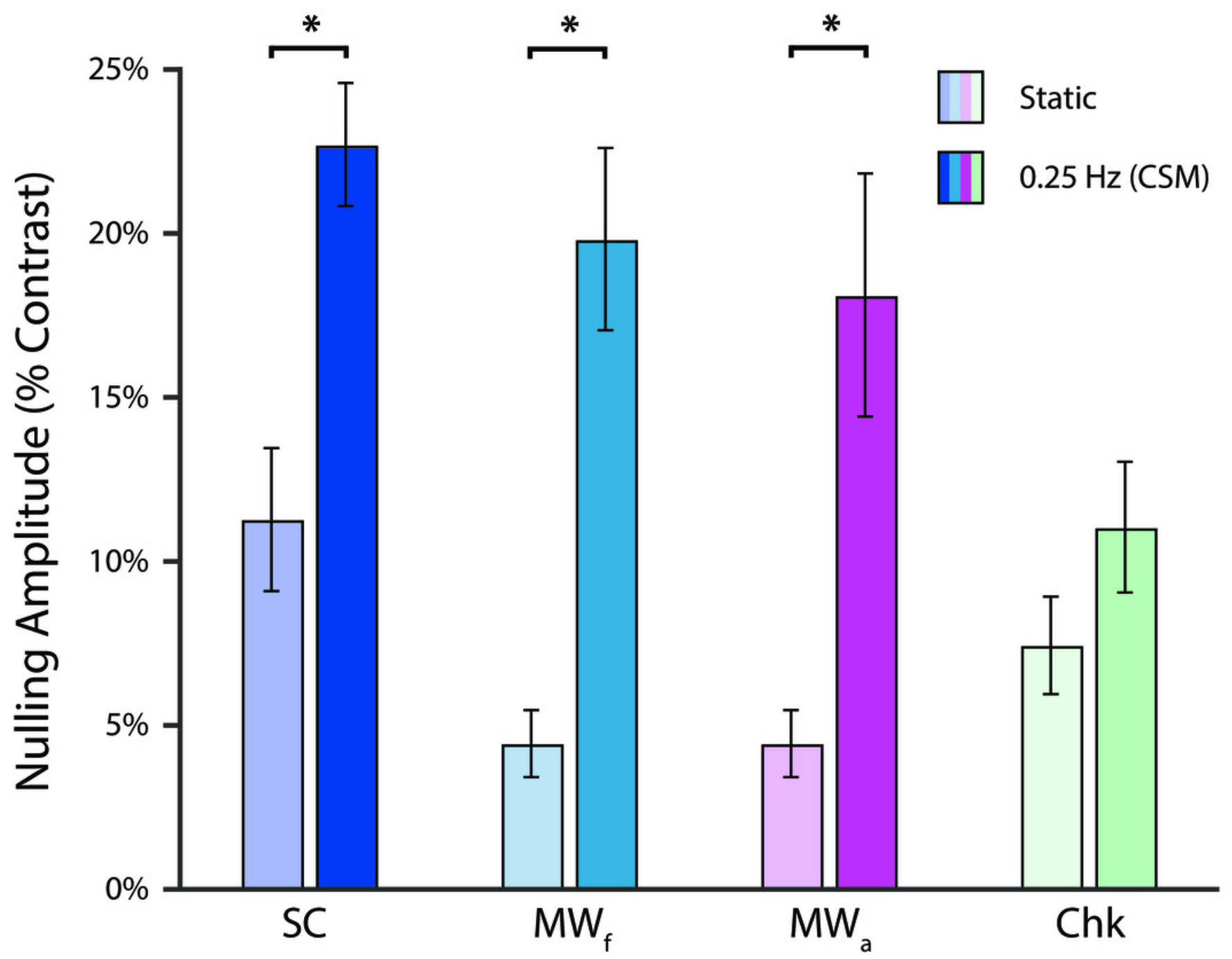




\section{Figure 4}

Just Noticeable Temporal Modulation (JNTM) limit

Illustration depicting lightness induction strength trends as stimulus modulation frequency approaches the limit at which continuous modulation of the stimulus can no longer be reliably detected by the observer, and is perceived to be static in nature, termed the Just Noticeable Temporal Modulation (JNTM) limit. Over increasingly slower temporal modulation frequencies, the lightness induction strength is hypothesized to change in three qualitative ways: induction strength will (a) continue to increase, (b) reach a plateau, or (c) monotonically decrease. An inflection point (d) for the latter two hypothetical paths could occur at any point between the lowest temporal modulation condition in this study $(0.25 \mathrm{~Hz})$ and the JNTM limit. Data points from the Simultaneous Contrast condition are shown in lowopacity for illustrative purposes only. 


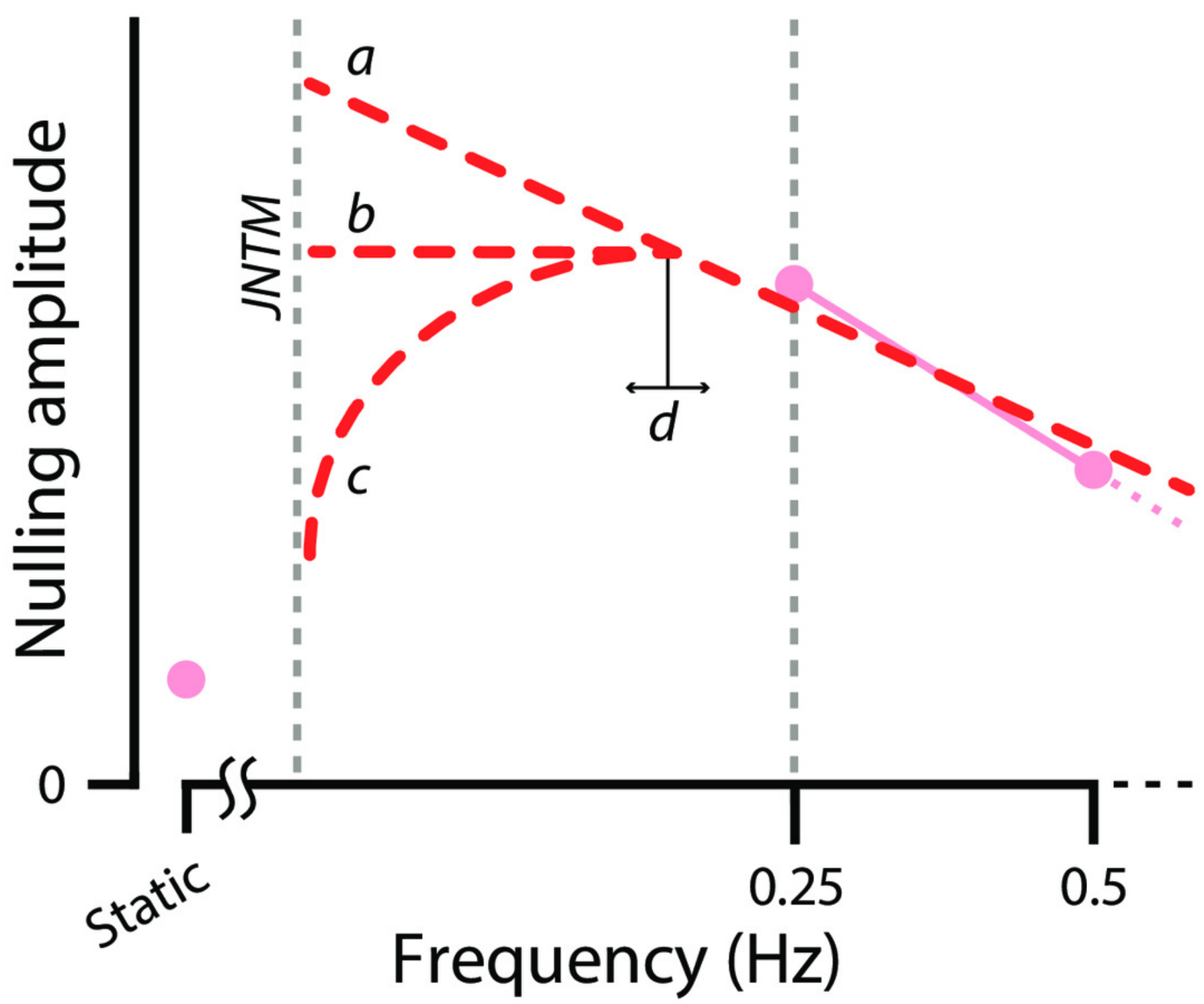

